ACCEPTED MANUSCRIPT

\title{
Comprehensive study on the concept of spectral-domain reflection and refraction
}

To cite this article before publication: Chunxiang Zhang et al 2019 Appl. Phys. Express in press https://doi.org/10.7567/1882-0786/ab4224

\section{Manuscript version: Accepted Manuscript}

Accepted Manuscript is "the version of the article accepted for publication including all changes made as a result of the peer review process, and which may also include the addition to the article by IOP Publishing of a header, an article ID, a cover sheet and/or an 'Accepted Manuscript' watermark, but excluding any other editing, typesetting or other changes made by IOP Publishing and/or its licensors"

This Accepted Manuscript is @ 2019 The Japan Society of Applied Physics.

During the embargo period (the 12 month period from the publication of the Version of Record of this article), the Accepted Manuscript is fully protected by copyright and cannot be reused or reposted elsewhere.

As the Version of Record of this article is going to be / has been published on a subscription basis, this Accepted Manuscript is available for reuse under a CC BY-NC-ND 3.0 licence after the 12 month embargo period.

After the embargo period, everyone is permitted to use copy and redistribute this article for non-commercial purposes only, provided that they adhere to all the terms of the licence https://creativecommons.org/licences/by-nc-nd/3.0

Although reasonable endeavours have been taken to obtain all necessary permissions from third parties to include their copyrighted content within this article, their full citation and copyright line may not be present in this Accepted Manuscript version. Before using any content from this article, please refer to the Version of Record on IOPscience once published for full citation and copyright details, as permissions will likely be required. All third party content is fully copyright protected, unless specifically stated otherwise in the figure caption in the Version of Record.

View the article online for updates and enhancements. 


\section{Comprehensive study on the concept of spectral-domain reflection and refraction}

Chunxiang Zhang, ${ }^{1,3}$ Zhixiang Deng, ${ }^{2}$ Yu Chen, ${ }^{2, *}$ Jun Liu, ${ }^{1,3}$ Thomas Davenport, ${ }^{3}$ and Dianyuan Fan ${ }^{2}$

${ }^{1}$ Laboratory for Micro-/Nano- Optoelectronic Devices of Ministry of Education, IFSA Collaborative Innovation Center, School of Physics and Electronics, Hunan University, Changsha 410082, China

${ }^{2}$ International Collaborative Laboratory of 2D Materials for Optoelectronics Science and Technology, Key Laboratory of Optoelectronic Devices and Systems of Ministry of Education and Guangdong Province, College of Optoelectronic Engineering, Shenzhen University, Shenzhen 518060, China

${ }^{3}$ Aston Institute of Photonic Technologies, School of Engineering and Applied Science, Aston University, Birmingham B4 7ET, U.K.

E-mail: yuchen@szu.edu.cn

Space-time duality and time-to-frequency mapping bring a lot of analogies. We apply the concept of optical reflection and refraction, for the first time, to the spectral domain. The optical reflection and refraction phenomenon are addressed in the spectral domain across a dispersion discontinuity boundary, where a weak probe pulse is co-propagating with a strong perpendicularly polarized parabolic pulse. The total internal reflection behavior in the spectral domain is analytically determined. Our results can complete the theory of the optical wave confinement in the spatial, temporal and spectral domains, which can be a guide for the spectrum engineering and characterization. 
Template for APEX (Jan. 2014)

Space and time are dual variables. The theory in space is possible to find its analogy in time and vice versa. Traditional reflection and refraction phenomena of optical waves at the interface between two homogeneous transparent media, which are described by the Snell's law and Fresnel formulas, have been known for many years and are always one of the important topics in physics textbooks [1, 2]. The essential prerequisite to observe these phenomena is that the light beam encounters a boundary where the refractive index changes abruptly. From the fundamental physics perspective, the sudden variation of the refractive index breaks the translational symmetry in space, which can vary the momentum of the photons while maintaining their energy across the boundary [3-5]. Significantly, the principle of the optical reflection and refraction is not limited to the spatial cases. Recently, the temporal reflection and refraction behaviors and analogies have been investigated based on the mathematical symmetry between diffraction and dispersion to achieve the adiabatic frequency conversion of ultrafast signals on picosecond timescales [6-12]. The frequency shifts experienced by the reflected and refracted pulses are determined by the frequency-dependent dispersion of materials in which the pulses are propagating $[6,7]$. Considering two temporal boundaries that satisfy the total internal reflection (TIR) condition, temporal waveguides can be constructed to confine the pulses within a fixed time window in which an optical pulse bounces back and forth between them [7, 8]. These results also provided a new method of manipulating the optical pulse via a pump-probe configuration in which the cross-phase modulation can be used to produce a moving temporal boundary [13-15].

In view of the time-to-frequency mapping associated with optical Fourier transformation technique [16], the optical reflection and refraction phenomenon in terms of the spectral domain, which is also possible, have received little attention. Actually, the temporal phase of a parabolic pulse imposed by the phase modulator based on a time lens [17] or through the cross-phase modulation (XPM) is equivalent to the dispersion effect which can also affect the temporal propagation of an optical pulse [18]. The XPM effect can impose an additional phase shift to the incident pulse, the value of which is proportional to the intensity of the other co-propagating pulse [19]. Considering this, a certain spectral shift can be required for the probe pulse through the desired $\mathrm{T}^{2}$-dependent phase resulting from the XPM of the parabolic pump pulse. A natural question then arises: Does the spectral analog of reflection 
and refraction of optical waves exist when the frequency shift of optical pulse crosses an interface with dispersion discontinuity? It is the aim of this paper to address this question both numerically and analytically.

In this paper, we show that, analogous to the common wisdom that considers both the reflection and refraction phenomena existing at boundaries formed by media with different refractive indices, the spectral analog of the reflection and refraction behaviors can also be realized as the spectrum of optical pulses shifts and encounters a spectral region where the refractive index changes suddenly. The TIR phenomenon is observed across a dispersion discontinuity boundary with a sufficiently high effective index change. The formation of spectral optical waveguide is possible to confine the optical pulse within a frequency interval where the TIR condition is satisfied across the two selected dispersion discontinuity boundaries.

The quadratic temporal phase shift can cause a translation in the spectrum. For a XPM-based time lens, a parabolic pump pulse can produce a time-dependent phase shift through the nonlinear Kerr effect [20]. We consider a situation where a weak probe pulse co-propagates with a strong perpendicularly polarized, negatively chirped parabolic pulse. The nonlinear propagation of an optical pulse can be modeled based on the coupled nonlinear Schrödinger equation (CNLSE) inclúding only nonlinear Kerr effect that can be numerically solved in an ultra-short pulse model with a split-step scheme in the symmetrical manner [19]. Given the very short propagation distances considered $(\sim \mathrm{mm})$, neither the loss nor the dispersion effects will play a significant role during the pulse propagation. We make an assumption that the negatively chirped parabolic pulse is generated by the fiber Bragg gratings or the pulse shaper:

$$
A_{\text {parabol }}(T)=\left\{\begin{array}{cc}
\sqrt{P_{p}} \sqrt{1-\frac{T^{2}}{T_{p}^{2}}} e^{i \alpha T^{2}} & -1 \leq T / T_{p} \leq 1 \\
0 & \text { otherwise }
\end{array}\right. \text {, }
$$

where $P_{p}$ is the peak power, $T_{p}$ is the FWHM duration of the pump pulse $\left|A_{\text {parabol }}(T)\right|^{2}$, and $\alpha$ is the chirp parameter. Assuming that the peak power of the probe wave is very weak compared to the negatively chirped parabolic pulse, the self-phase modulation of the probe wave can be neglected, and then the propagation of the probe wave can be described by the following NLSE 
Template for APEX (Jan. 2014)

$$
\frac{\partial A(z, T)}{\partial z}=i \gamma\left|A_{\text {parabol }}(z, T)\right|^{2} A(z, T),
$$

where $\gamma$ is the fiber nonlinear parameter. For simplicity we consider a transform-limited parabolic field intensity $\left|A_{\text {parabol }}(T)\right|^{2}=P_{p}\left(1-T^{2} / T_{p}^{2}\right)$. The NLSE described by Eq.(2) becomes [20]

$$
\frac{\partial A(z, T)}{\partial z}=\frac{i}{L_{n l}} A(z, T)-\frac{i T^{2}}{L_{n l} T_{p}^{2}} A(z, T)
$$

where $L_{n l}=1 /\left(\gamma P_{p}\right)$ is the nonlinear length of the strong pump pulse. This results in a transfer function, which is the impulse response of a parabolic phase modulated element, and equals to

$$
h_{T}(T)=\exp \left[i\left(\frac{1}{L_{n l}}-\frac{T^{2}}{L_{n l} T_{p}^{2}}\right) z\right] .
$$

The function has the same complex quadratic differential structure as the dispersion effect. In the transform domain the quadratic phase modulation and second-order dispersion are the dual of each other. This means that the same phenomena related to dispersion can be seen in the frequency domain because of the phase transformation associated with XPM. To put simply, a XPM-based time lens can broaden or narrow the spectrum of an optical pulse, or vary the center frequency while maintaining the pulse shape.

It is generally known that the optical reflection and refraction are observed at an interface when the refractive index $n(\omega)$ changes on the spatial scale of the optical wavelength $[1,2,4]$. To achieve the scattering of the weak probe wave off the boundary with an abrupt refractive index variation, we consider the limiting case of a sharp refractive index change, i.e., $n(\omega)=n_{1}(\omega)+\Delta n$ with $|\Delta n|<<n_{1}(\omega)$. The propagation constant $\beta$ (i.e., effective mode index) of the waveguides is assumed to vary across the discontinuous boundary to describe a potential step, taking the two asymptotic values $\beta_{1}\left(\omega<\omega_{\mathrm{B}}\right)$ and $\beta_{2}\left(\omega>\omega_{\mathrm{B}}\right)$. $\omega_{\mathrm{B}}$ is the frequency detuning between the optical pulse and the boundary frequency position. In the nearest-neighbor and tight-binding approximations [21], the envelope evolution of the weak probe wave is described by the following equation in the time domain:

$$
\frac{\partial A(z, T)}{\partial z}=\frac{i}{L_{n l}} A(z, T)-\frac{i T^{2}}{L_{n l} T_{p}^{2}} A(z, T)+i \mathrm{~F}^{-1}[\Delta \beta(\omega) A(z, \omega)],
$$

where Adenotes the complex field envelop of the weak probe wave and $A$ is its Fourier 


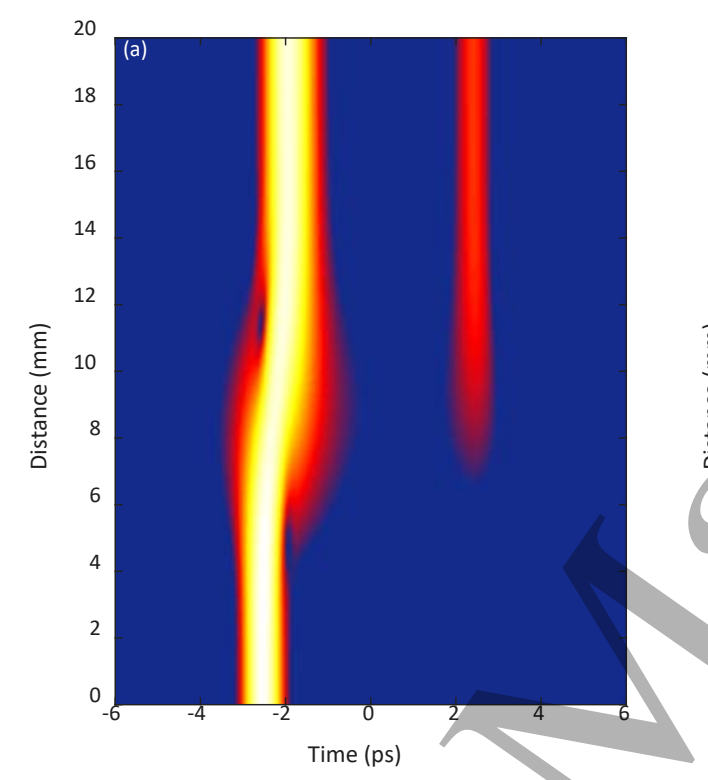

Template for APEX (Jan. 2014)

transformation, $\mathrm{F}^{-1}$ stands for the inverse of Fourier transformation, and $\Delta \beta(\omega)=\beta_{B} H\left(\omega-\omega_{B}\right)$ is the propagation constant mismatch, which describes a sharp potential step. $\beta_{B}=\beta_{2}-\beta_{1}$ is the change in the propagation constant caused by a sudden index change $\Delta n$. The Heaviside function $H\left(\omega-\omega_{B}\right)$ takes a value of 0 for $\omega<\omega_{B}$ and 1 for $\omega>\omega_{B}$. The parameters in the following simulation are taken as $\omega_{\mathrm{B}}=2 \mathrm{THz}, T_{p}=5 \mathrm{ps}, P_{p}=95 \mathrm{~W}$, and $\gamma=0.1(\mathrm{~W} \cdot \mathrm{m})^{-1}$.

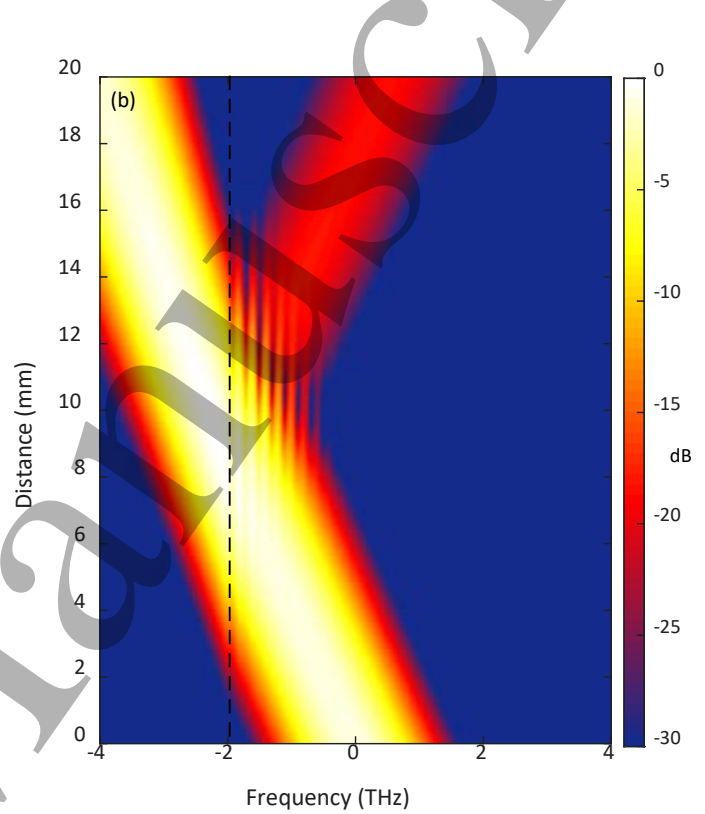

Fig. 1. Observation of the spectral reflection and refraction at a dispersion discontinuity boundary, and the occurrence of the corresponding pulse-splitting in the temporal domain. Density maps of the temporal (a) and corresponding spectral (b) evolutions of an optical pulse in the presence of a dispersion discontinuity boundary. The dashed black line depicts the interface of the sharp index step ( $\beta=\beta_{1}$ and $\beta=\beta_{2}$ correspond to the propagation constants in the left and right regions of the dashed lines, respectively).

An example of the spectral reflection and refraction for an optical pulse at a dispersion discontinuity boundary $\left[\beta_{B}=0.6 \mathrm{~mm}^{-1}\right]$ caused by a sudden index change is shown in Fig.1, which illustrates the (a) temporal and (b) spectral evolutions of a weak probe pulse with a Gaussian profile. For the following numerical simulations, the peak power and width of the probe wave are set to $1 \mathrm{~W}$ and $0.25 \mathrm{ps}$, respectively, and its time delay relative to the strong pump pulse is 2.5 ps. Clearly, the wave packet in the frequency domain is partially reflected and partially transmitted. As can be seen from Fig. 1(a), the incident wave packets can be broken into both reflected and transmitted pulses that are propagating at different moving 
Template for APEX (Jan. 2014)

velocities. The variation of the moving velocities is what leads to the apparent bending in the frequency domain in Fig. 1(b). This frequency shift is attributed to the velocity change as a result of the sharp change of the refractive index, which ultimately result in the spectral analogue of the reflection and refraction phenomena for optical pulses.
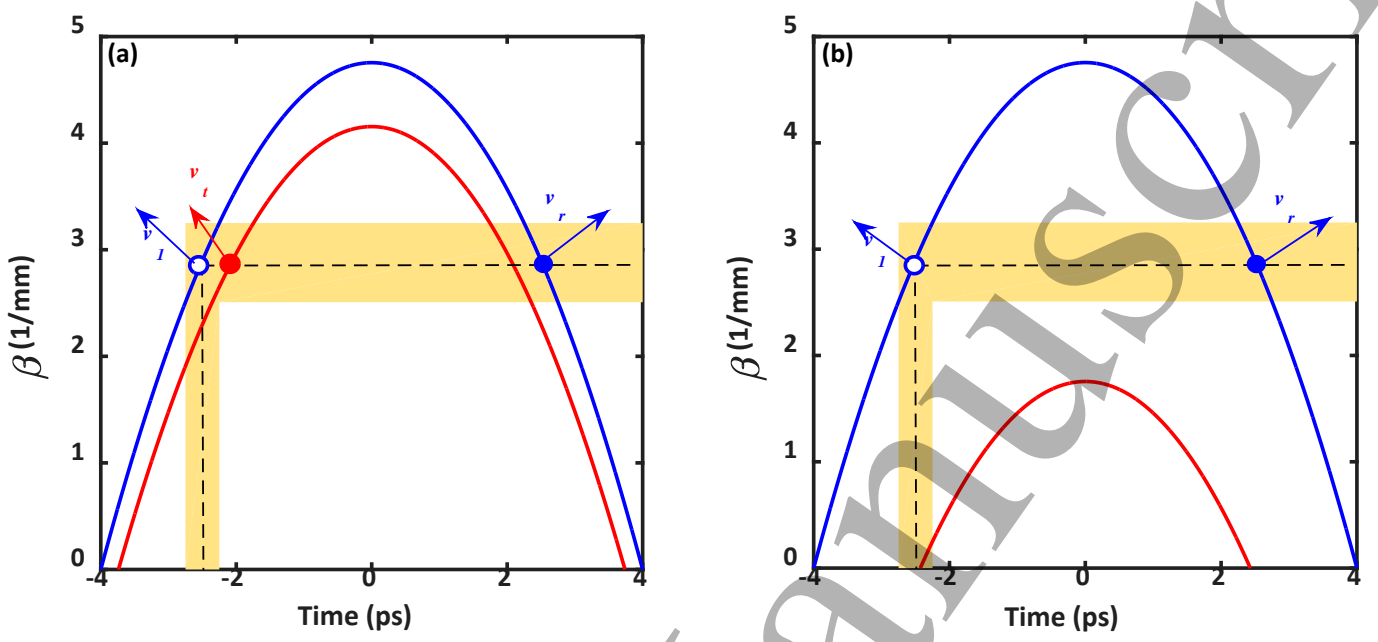

Fig. 2. Temporal phase curves $\beta(T)$ of the two frequency regions far from the interface [ $\omega<\omega_{B}$ (blue solid line) and $\omega>\omega_{\mathrm{B}}$ (red solid line)] for (a) $\beta_{\mathrm{B}}<\beta_{\mathrm{c}}$ and (b) $\beta_{\mathrm{B}}>\beta_{\mathrm{c}}$, where $\beta_{\mathrm{c}}$ is the critical value of spectral TIR (refractionless propagation) of an optical pulse. The shaded region shows the temporal extent of an incident weak probe pulse and corresponding range of propagation constants for $\omega<\omega_{B}$. The open circles correspond to the initial temporal position $T_{1}$ of the weak probe wave relative to the strong pump pulse. The temporal position $T_{2}$ of the reflected and refracted pulse, obtained from the phase-matching relation, i.e. $\beta\left(T_{1}\right)=$ $\beta\left(T_{2}\right)$, are indicated by filled blue and red circles, respectively. $v_{1}, v_{r}$ and $v_{t}$ denote the frequency-shifted velocities of the incident, reflected, and refracted pulses in the $f$-z plane.

By including the nonlinear propagative phase and allowing for a dispersion discontinuity boundary, the refraction and reflection illustrated by Snell's Law can be expanded to the frequency domain. Far from the dispersion discontinuity boundary, i.e., for $\omega<<(>>) \omega_{\mathrm{B}}$, the scattering solutions to Eq. (4) are plane waves $A(z, T): \exp [i \omega T+i \beta(T) z]$, where the temporal phase profiles are given by $\beta(T)=\left(1-T^{2} / T_{p}^{2}\right) / L_{n l}$ for $\omega<\omega_{B}$ and $\beta(T)=\left(1-T^{2} / T_{p}^{2}\right) / L_{n l}+\beta_{B}$ for $\omega>\omega_{B}$. We can see that the two temporal phase curves exhibit the same profiles but displaced by the amount $\beta_{B}$. To observe the spectral refraction, the two temporal phase curves are required to be partially overlapped, i.e., the constraint $L_{n l} \cdot\left|\beta_{B}\right|<1$ must be satisfied; otherwise, 
the spectral analogue of the TIR can be observed for any incident wave packet. For the left incidence side, we consider an incident wave packet with a relative time delay $T_{1}\left(0<T_{1}<T_{p}\right)$, which propagates longitudinally along the fiber with a group velocity $v_{i}=(\partial \beta / \partial T)_{T=T_{1}}=-2 T_{1} /\left(T_{p}^{2} L_{n l}\right)<0$. The refracted and reflected wave packets haye a shifted relative time delay $T_{2}, T_{3}$ respectively, which are determined from the following temporal phase matching rule, i.e.,

$$
\begin{gathered}
\left(1-T_{2}^{2} / T_{p}^{2}\right) / L_{n l}=\left(1-T_{1}^{2} / T_{p}^{2}\right) / L_{n l}+\beta_{B}, \\
\left(1-T_{3}^{2} / T_{p}^{2}\right) / L_{n l}=\left(1-T_{1}^{2} / T_{p}^{2}\right) / L_{n l} .
\end{gathered}
$$

With the constraint $v_{t}^{-1}=-2 T_{2} /\left(T_{p}^{2} L_{n l}\right)<0$ and $v_{r}^{-1}=-2 T_{3} /\left(T_{p}^{2} L_{n l}\right)>0$ [Fig. 2(a)], the solutions of the quadratic equation (6) and (7) are

$$
T_{2}=T_{1} \cdot \sqrt{1-\frac{T_{p}^{2} \beta_{B} L_{n l}}{T_{1}^{2}}} \text { and } T_{3} \neq-T_{1} \text {. }
$$

These solutions stand for the refracted and reflected temporal positions and correspond to the red and blue points in Fig.2(a). The refracted wave packet, propagating with a longitudinal frequency-shifted velocity $v_{t}<0$ that is generally different to $v_{1}$, yields a bending of the transmitted pulse (spectral refraction).

As seen from Eq. (8), the relative time shift $T_{2}$ of the refracted pulse depends on the magnitude of $\beta_{\mathrm{B}}$. We need to check what happens if $\beta_{\mathrm{B}}$ is large enough that the solution $T_{2}$ of the Eq. (6) loses its physical meaning. No refracted pulse can form across the dispersion discontinuity boundary and the incident pulse must be totally reflected. This is the spectral analog of the well-known phenomenon of TIR. The condition for the spectral TIR is found from Eq. (8) to be

$$
\beta_{B}>\beta_{c}=T_{1}^{2} /\left(T_{P}^{2} \cdot L_{n l}\right) .
$$

The spectral TIR can also be understood from the two temporal phase curves in Fig. 2(b), where the red curve does not intersect with the shaded region. Figure 3 shows the corresponding numerical results under $\beta_{B}=3 \mathrm{~mm}^{-1}$. In this situation, the transmitted phase curve locates below the shaded region. As predicted by the above theory, there is no transmitted pulse (refractionless propagation), and the wave packet, possessing the same frequency-shifted velocity as the incident wave packet but reversed in sign, is fully reflected. It is not difficult to observe from Fig. 3 that the refracted pulse is spectrally narrowed and 
Template for APEX (Jan. 2014)

temporally broadened which is attributed to the fact that the shaded region on the transmitted curve in Fig. 2(b) covers a much wider temporal region based on the phase-matching relation for all positions in the temporal domain.
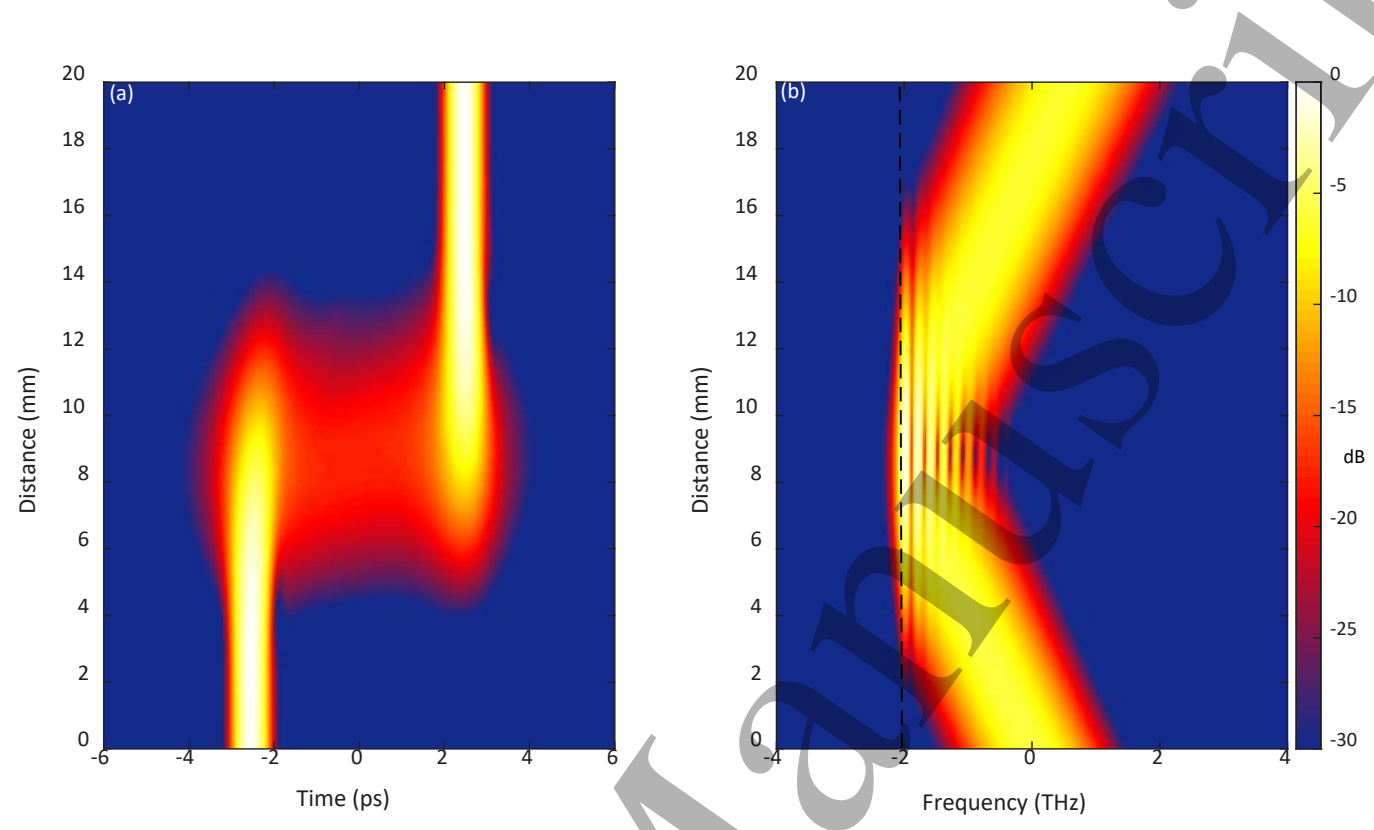

Fig. 3. Spectral analog of TIR phenomenon. Temporal (a) and spectral (b) evolutions of an optical pulse undergoing TIR at a dispersion discontinuity boundary located at $2 \mathrm{THz}$ for $\beta_{\mathrm{B}}=3 \mathrm{~mm}^{-1}$. The incident pulse is almost completely reflected, and the reflected pulse has the same frequency-shifted velocity as the incident pulse but reversed in sign.

We can extend the concept of a spatial optical waveguide to the spectral domain based on the above results with the spectral TIR phenomenon. If two dispersion discontinuity boundaries for TIR are set, it is natural to conclude that the optical pulse will be confined within the frequency interval. For example, Figure 4 shows the numerical observation of a weak probe pulse trapped within a rectangular scattering potential [ $\Delta \beta=0$ for $|\omega|<\omega_{B}, \quad \Delta \beta=\beta_{B}$ for $|\omega|>\omega_{B}$ ] formed by two dispersion discontinuity boundaries. The optical pulse bouncing in the frequency domain is accompanied by a back-and-forth temporal conversion of the weak probe pulse [see Fig. 4(a)]. This behavior is strikingly similar to that of an optical beam inside two spatial boundaries. Therefore, we can refer to the configuration with two dispersion discontinuity boundaries as a spectral waveguide. Like the spatial and temporal waveguide $[7,8]$, the concept of the spectral waveguide proposed here can be potentially implemented for all-optical signal processing, such as the pulse self-imaging which can 
duplicate a single pulse into multiple identical pulses in the frequency domain.

Among these effects, the local variation of the dispersion occurs within the dispersion profile comparable to the spectral scale of the order of the optical wavelength, which is experimentally challenging. An alternative to create the dispersion discontinuity boundary is through achieving the avoided-mode crossing process, which is similar to the situation in Ref. [22]. In this case, the nonlinear mode coupling between different modes can produce an avoided-mode crossing, altering its local dispersion profile. It is important to note here that instead of modeling the actual coupling between the weak probe pulse and the strong parabolic pulse the model Eq. (5) simply shifts the mode frequencies of the probe pulse in a way that is typical for an avoided-mode crossing. Therefore, it makes sense to consider a single mode family in the simulations only. The related experimental implementation will be a subject for future studies.

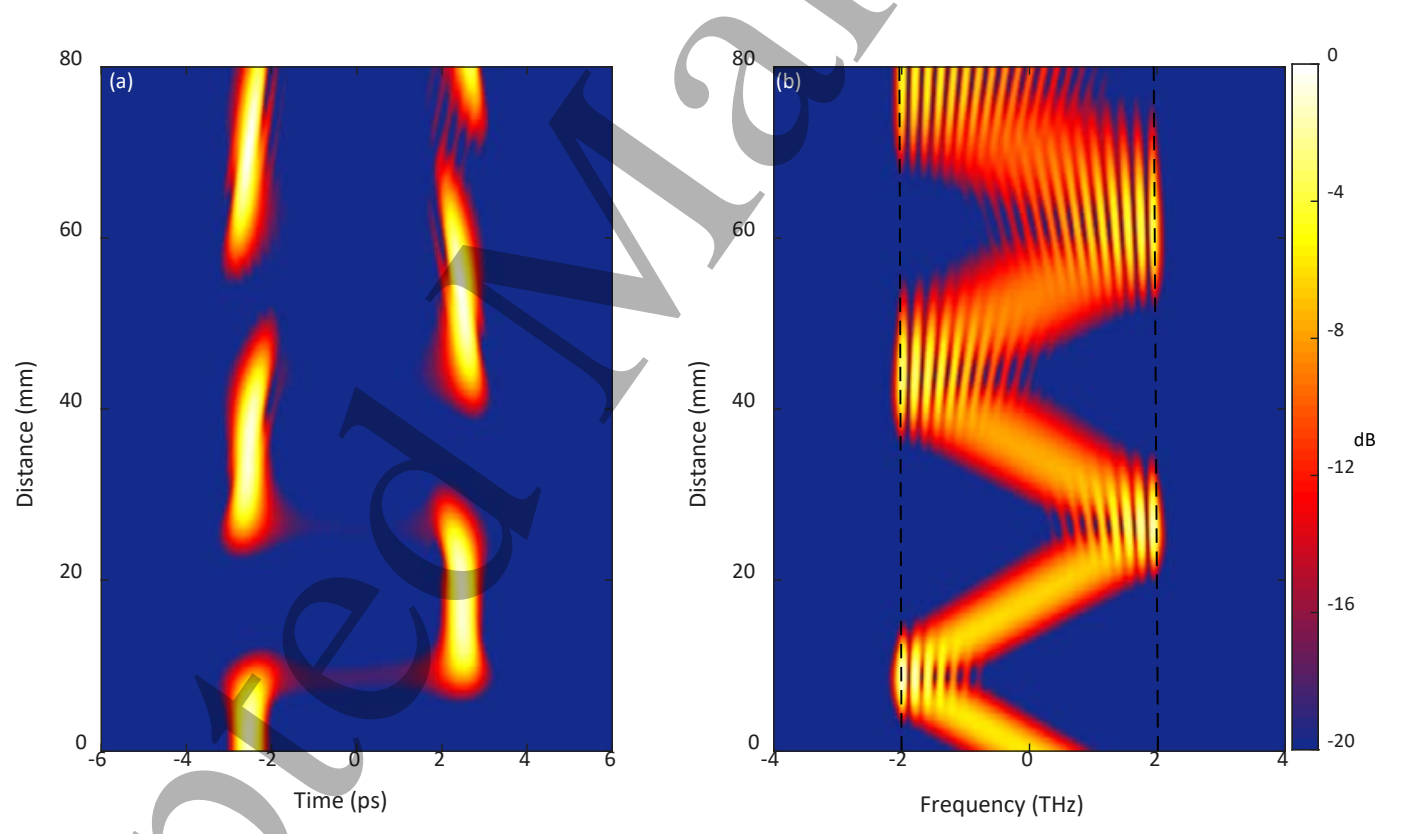

Fig. 4. Simulations of the spectral waveguides for optical pulses. Evolutions of the (a) temporal shape and (b) spectrum of a Gaussian pulse inside a $4 \mathrm{THz}$ wide spectral waveguide. Dashed horizontal lines show the spectral boundaries that form the waveguide core.

In conclusion, we have proposed an alternative approach to investigate the spectral-domain refraction and reflection phenomena, which is the analogy of their spatial or temporal counterpart. By considering a weak probe pulse co-propagating with a strong 
Template for APEX (Jan. 2014)

perpendicularly polarized parabolic pulse, we have been able to obtain the spectral shift experienced by a weak probe wave when the two pulses interact with each other through the nonlinearity. Our numerical simulation reveals that, across the dispersion discontinuity boundary, a spectral analog of an optical refraction and reflection exists. Furthermore, the analytical derivation of the nonlinear phase matching rule reveals that the spectral TIR can be achieved by adjusting the displacement of the dispersion discontinuity. Our findings demonstrate that the optical reflection and refraction are in no way exclusively associated with linear propagation; it is a general feature of wave confinement in some direct or inverse coordinate, regardless of spatial, temporal or spectral domains.

\section{Acknowledgments}

National Natural Science Fund Foundation of China (NSFC) (61875132, 61805115, and 61605166), and Shenzhen Government' s Plan of Science and Technology (JCYJ20170302153731930). 
Template for APEX (Jan. 2014)

\section{References}

1) M. Born, and E. Wolf, Principles of Optics: Electromagnetic Theory of Propagation, Interference, and Diffraction of Light, $7^{\text {th }}$ expanded ed. (Cambridge University Press, Cambridge, England, 1999).

2) B. E. A. Salesh and M. C. Teich, Fundamentals of Photonics, $2^{\text {nd }}$ ed. (Wiley-Interscience, Hoboken, NJ, 2007).

3) N. Yu, P. Genevet, M. A. Kats, F. Aieta, J. P. Tetienne, F. Capasso, and Z. Gaburro, "Light propagation with phase discontinuities: generalized laws of reflection and refraction,” Science, 334(21), 333-337 (2011).

4) A. Akbarzadeh, N. Chamanara, and C. Caloz, "Inverse prism/based on temporal discontinuity and spatial dispersion,” Opt. Lett. 43(14), 3297-3300, (2018).

5) S. Longhi, "Reflectionless and invisible potentials in/photonic lattices,” Opt. Lett. 42(16), 3229-3232, (2017).

6) B. W. Plansinis, W. R. Donaldson, and G. P. Agrawal, "What is the temporal analog of reflection and refraction of optical beams?,” Phys. Rev. Lett. 115(18), 183901 (2015).

7) B. W. Plansinis, W. R. Donaldson, and G. P. Agrawal, “Temporal waveguides for optical pulses,” J. Opt. Soc. Am. B 33(6), 1112-1119 (2016).

8) J. Zhou, G. Zheng, and J. Wu, “Comprehensive study on the concept of temporal optical waveguides,” Phys. Rev. A 93(6), 063847 (2016).

9) G. A. Menendez, and B. Maes, “Time reflection and refraction of graphene plasmons at a temporal discontinuity,” Opt. Lett. 42(23), 5006-5009 (2017).

10) B. W. Plansinis, W. R. Donaldson, G. P. Agrawal, "Spectral splitting of optical pulses inside a dispersive medium at a temporal boundary,” IEEE J. Quantum Electron. 52(12), 1-8 (2016).

11) H. Chen, C. Qin, B. Wang, P. Lu, "Discrete refraction and reflection in temporal lattice heterostructures,” Opt. Lett. 44(2), 363-366 (2019).

12) M. Petev, N. Westerberg, D. Moss, E. Rubino, C. Rimoldi, S. L. Cacciatori, F. Belgiorno and D. Faccio, "Blackbody emission from light interacting with an effective moving dispersive medium,” Phys. Rev. Lett. 111(4), 043902 (2013).

13) S. F. Wang, A. Mussot, M. Conforti, X. L. Zeng, and A. Kudlinski, "Bouncing of a dispersive wave in a solitonic cage,” Opt. Lett. 40(14), 3320-3323 (2015). 
Template for APEX (Jan. 2014)

14) Z. X. Deng, J. Liu, X. W. Huang, C. J. Zhao, and X. L. Wang, “Active control of adiabatic soliton fission by external dispersive wave at optical event horizon,” Opt. Express 25(23), 28556-28566 (2017).

15) I. Oreshnikov, R. Driben, and A. V. Yulin, "Interaction of high-order solitons with external dispersive waves,” Opt. Lett. 40(23), 5554-5557(2015).

16) G. Bosco, A. Garena, V. Curri, R. Gaudino, P. Poggiolini, and S. Benedetto, "Suppression of spurious tones induced by the Split-step method in fiber systems simulation,” IEEE Photon. Technol. Lett. 12(5), 489-491, (2000).

17) B. H. Kolner and M. Nazarathy, “Temporal imaging with a time lens,” Opt. Lett. 14(12), 630-632 (1989).

18) E. R. Andresen, C. Finot, D. Oron, and H. Rigneault, "Spectral analog of the Gouy phase shift,” Phys. Rev. Lett. 110(14), 143902 (2013).

19) G. P. Agrawal, Nonlinear Fiber Optics (Academic Press, San Diego, 2001).

20) T. T. Ng, F. Parmigiani, M. Ibsen, Z. Zhang, P. Petropoulos, and D. J. Richardson, "Compensation of linear distortions by using XPM with parabolic pulses as a time lens," IEEE Photon. Tech. Lett. 20(13), 1097-1099 (2008).

21) T. B. Boykin, and G. Klimeck, “The discretized Schrödinger equation and simple models for semiconductor quantum wells,” Eur. J. Phys. 25(4), 503-514 (2004).

22) S. Ramelow, A. Farsi, S. Clemmen, J. S. Levy, A. R. Johnson, Y. Okawachi, M. R. E. Lamont, M. Lipson, and A. L. Gaeta, "Strong polarization mode coupling in microresonators," Opt. Lett. 39(17), 5134-5137 (2014) 
Template for APEX (Jan. 2014)

\section{Figure Captions}

Fig. 1. Observation of the spectral reflection and refraction at a dispersion discontinuity boundary, and the occurrence of the corresponding pulse-splitting in the temporal domain. Density maps of the temporal (a) and corresponding spectral (b) evolutions of an optical pulse in the presence of a dispersion discontinuity boundary. The dashed black line depicts the interface of the sharp index step $\left(\beta=\beta_{1}\right.$ and $\beta=\beta_{2}$ correspond to the propagation constants in the left and right regions of the dashed lines, respectively).

Fig. 2. Temporal phase curves $\beta(T)$ of the two frequency regions far from the interface [ $\omega<\omega_{\mathrm{B}}$ (blue solid line) and $\omega>\omega_{\mathrm{B}}$ (red solid line)] for (a) $\beta_{\mathrm{B}}<\beta_{\mathrm{c}}$ and (b) $\beta_{\mathrm{B}}>\beta_{\mathrm{c}}$, where $\beta_{\mathrm{c}}$ is the critical value of spectral TIR (refractionless propagation) of an optical pulse. The shaded region shows the temporal extent of an incident weak probe pulse and corresponding range of propagation constants for $\omega<\omega_{B}$. The open circles correspond to the initial temporal position $T_{1}$ of the weak probe wave relative to the strong pump pulse. The temporal position $T_{2}$ of the reflected and refracted pulse, obtained from the phase-matching relation, i.e. $\beta\left(T_{1}\right)=$ $\beta\left(T_{2}\right)$, are indicated by filled blue and red circles, respectively. $v_{1}, v_{r}$ and $v_{t}$ denote the frequency-shifted velocities of the incident, reflected, and refracted pulses in the $f$-z plane.

Fig. 3. Spectral analog of TIR phenomenon. Temporal (a) and spectral (b) evolutions of an optical pulse undergoing TIR at a dispersion discontinuity boundary located at $2 \mathrm{THz}$ for $\beta_{\mathrm{B}}=3 \mathrm{~mm}^{-1}$. The incident pulse is almost completely reflected, and the reflected pulse has the same frequency-shifted velocity as the incident pulse but reversed in sign.

Fig. 4. Simulations of the spectral waveguides for optical pulses. Evolutions of the (a) temporal shape and (b) spectrum of a Gaussian pulse inside a $4 \mathrm{THz}$ wide spectral waveguide. Dashed horizontal lines show the spectral boundaries that form the waveguide core. 

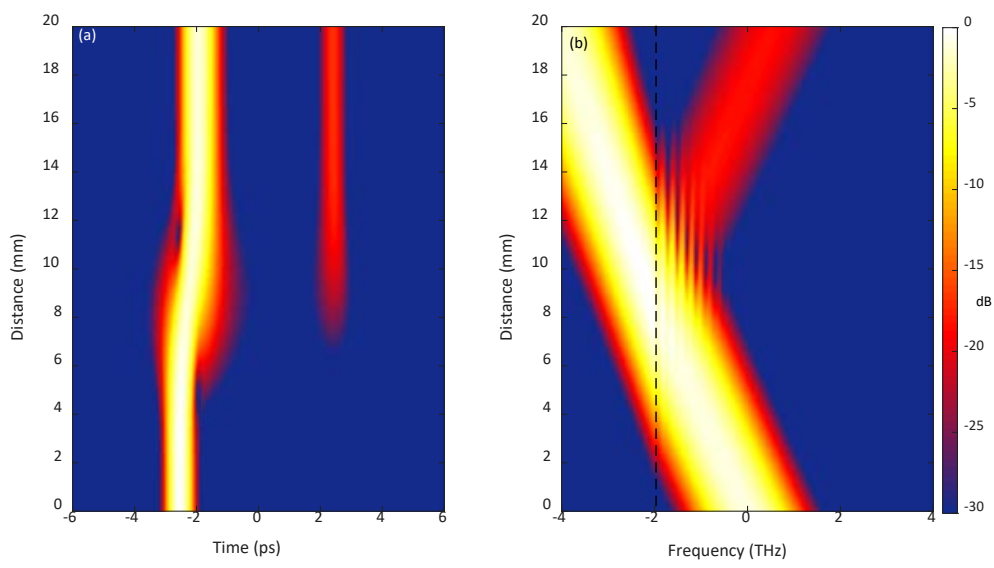

Fig.1.
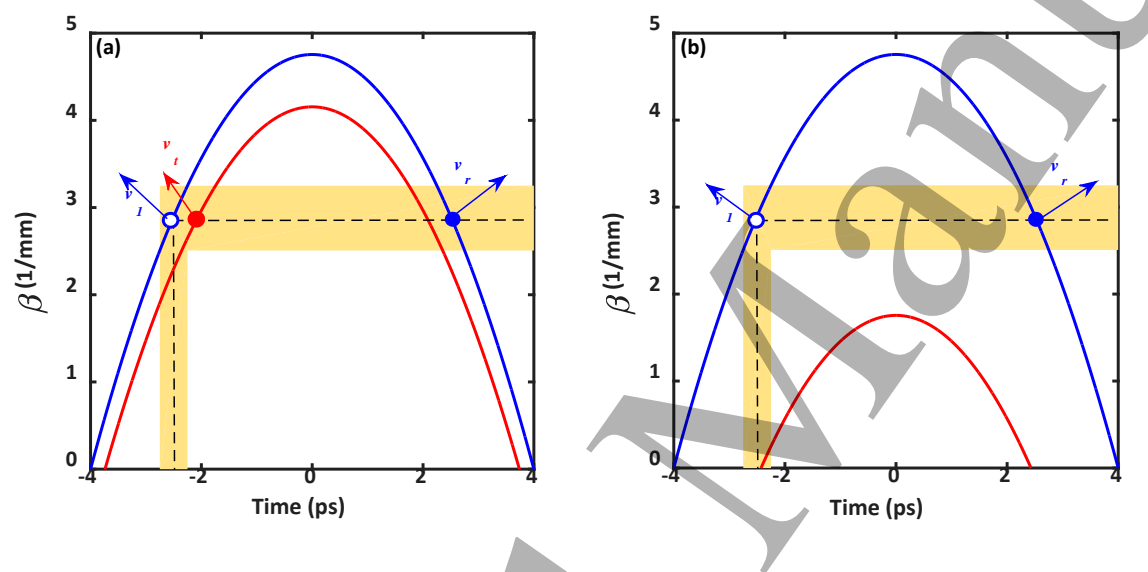

Fig. 2.
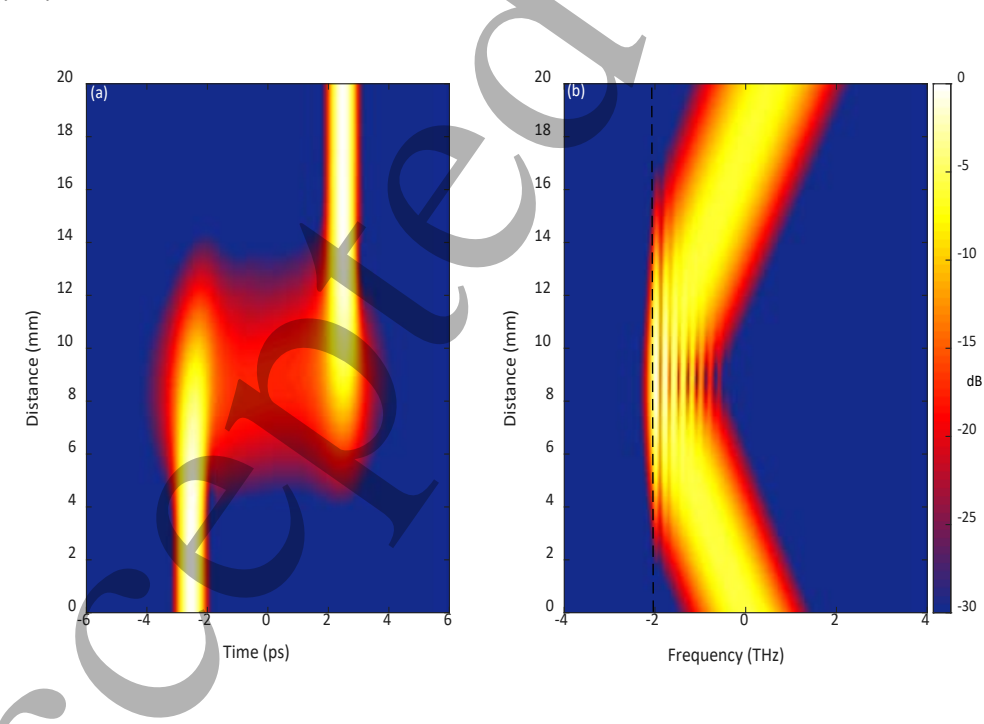

Fig. 3. 
